Universidad Autónoma de Chile, Departamento de Postgrado. Santiago, Chile. aKinesiólogo, Magister en Neurociencias. bPsicóloga. ${ }^{\mathrm{C} P h D .}$

Trabajo no recibió financiamiento. Los autotes declaran no tener conflictos de interés.

Recibido el 16 de octubre de 2017, aceptado el 12 de abril de 2018.

Correspondencia a: Germán Wikee Diagonal Travesía \#572, Pudahuel, Santiago. german.wikee@gmail.com

\section{Capacidad física y reserva cognitiva como factores protectores de las funciones atencionales en adultos mayores}

\author{
GERMÁN WIKEE ${ }^{a}$, DIANA MARTELLA ${ }^{\mathrm{b}, \mathrm{c}}$
}

Background: Attention is considered a central control mechanism in cognitive processing systems. Attention deficits contribute to the symptomatic profile of Alzheimer's disease (AD). Physical exercise and cognitive reserve, could delay cognitive impairment and constitute a protective factor against clinical manifestations of AD. Aim: To relate the functionality of the attentional networks with physical activity and cognitive reserve. Material and Methods: Three groups of 20 older adults each (control, physical activity and with osteoarthritis) were studied. The Functional capacity assessment test, Cognitive Reserve Questionnaire and the Attention Networks Test - for Interactions and Vigilance or ANTI-V were applied to participants. Results: Significant differences were observed in the response times of the alert, orientation and executive network, and the percentage of success in the network orientation and executive network. No differences between groups were observed for the different indicators of vigilance. Conclusions: These results confirm the benefits of physical exercise as a protective factor for attentional functioning.

(Rev Med Chile 2018; 146: 570-577)

Key words: Attention; Cognitive Aging; Cognitive Reserve; Physical Fitness.

\section{L} os cambios de las funciones cognitivas asociados a la edad no son uniformes, siendo la memoria y la atención las esferas cognitivas más afectadas ${ }^{1}$.

El Deterioro Cognitivo Leve (DCL) es un diagnóstico en revisión, considerado como un estado cognitivo transicional entre el envejecimiento normal y las primeras etapas de la demencia ${ }^{2}$. Los individuos con DCL presentan mayor riesgo de progresión a la enfermedad de Alzheimer (EA). Estudios sugieren que los déficits de atención se producen durante sus primeras etapas de la EA y contribuyen a su perfil sintomático ${ }^{3,4}$. Estas alteraciones en la función atencional puede ser un útil indicador para el diagnóstico, pronóstico y un punto potencial de intervención en personas con EA prodrómica ${ }^{1,6-11}$.

Según la Neurociencia Cognitiva, la atención puede ser considerada un componente central en el control del procesamiento cerebral. El modelo tripartido de Posner, considera a la atención como un sistema orgánico complejo compuesto de tres redes separadas anatómica y funcionalmente: la red ejecutiva o anterior, la red de orientación o posterior, y la red de vigilancia ${ }^{1,12-14}$.

Es importante destacar que las tres redes se pueden evaluar mediante el Attention Network Test (ANT), desarrollado por Posner y colabora- 
dores. El ANT original y sus versiones posteriores, desarrolladas para evaluar interacciones entre las redes $^{15,16}$, consisten en una combinación de tareas de pre-señalización y flancos propuestas por Posner (1980) y Eriksen \& Eriksen (1974) respectivamente ${ }^{17}$. El ANT ha demostrado su utilidad para evaluar la eficiencia de las redes atencionales no sólo en estudios conductuales ${ }^{7,8,16}$, sino también en neuroimagen ${ }^{18}$ y en pacientes con demencia o en sus etapas previas ${ }^{1,7,8,11}$. Además, la versión ANTI-Vigilance (ANTI-V) propuesta por Martella et al (2014), incluye la medición del componente tónico en la red de alerta (vigilancia). La tarea incluye ensayos donde los participantes deben detectar un pequeño e infrecuente cambio en la longitud en la flecha objetivo. Posteriormente, los datos se analizan mediante la aplicación de la Teoría de Detección de Señales (TDS), que indica la naturaleza del cambio en la vigilancia, y si depende de la habilidad discriminativa o el criterio de respuesta del participante ${ }^{7}$.

A nivel internacional destaca un interés creciente en el estudio de los factores relacionados al funcionamiento cognitivo en personas mayores, como son el estilo de vida, la interacción social, la nutrición y la actividad física ${ }^{19,20}$. Este último, se considera un factor relevante implicado en el retraso del deterioro cognitivo y la conservación de la inteligencia fluida. Asimismo, la actividad física se asocia a un envejecimiento cognitivo saludable, tanto en envejecimiento cerebral normal como en fases de deterioro cognitivo, siendo en un componente habitual en programas de promoción de salud en adultos mayores ${ }^{20-23}$.

Por otra parte, la reserva cognitiva (RC), descrita como la capacidad del cerebro para tolerar mejor los efectos de la patología asociada a la demencia, desarrollada como una capacidad innata o del efecto de las experiencias vividas, como la educación o la ocupación laboral ${ }^{24}$, y puede constituir un factor protector frente a la manifestación clínica de la EA ${ }^{24-27}$. La RC es modificable a través de la experiencia y el aprendizaje, donde la estimulación cognitiva se considera una actividad necesaria para fortalecer y mantener las conexiones neurales en la tercera edad ${ }^{26,27}$.

En base a estas evidencias, la actividad física y la reserva cognitiva representan factores protectores que potenciarían un envejecimiento cognitivo saludable, sin embargo, existe escasa evidencia que permita conocer el efecto acumulativo del ejercicio físico sobre el funcionamiento atencional, ya que los hallazgos actuales se relacionan a los efectos agudos del ejercicio sobre esta función cognitiva. En el caso de la reserva cognitiva, se desconoce su grado de influencia sobre las redes atencionales.

El objetivo de este estudio es evaluar la eficiencia funcional de las redes atencionales, en tres grupos de adultos mayores con distintos niveles de actividad física y reserva cognitiva.

\section{Material y Método}

\section{Participantes}

El estudio es no experimental comparativo de cohorte transversal ${ }^{28}$. Los participantes fueron 60 adultos mayores del Centro Deportivo Alamiro Correa de Pudahuel (Región Metropolitana de Chile). Todos fueron voluntarios y cumplieron los siguientes criterios de inclusión: Personas sanas mayores de 65 años; sin patologías o antecedentes de salud relevantes (Ejemplo: Cáncer, Insuficiencia Cardíaca, ACV); sin dificultades visuales o auditivas graves; que firmaran el consentimiento informado de participación en el estudio. Se conformaron 3 grupos de 20 sujetos, con las siguientes características: Grupo Artrosis, sujetos que participaban en talleres de actividad física terapéutica por gonartrosis leve o moderada; Grupo Actividad Física, formado por personas que realizaban actividad física permanente en el centro deportivo (mínimo 2 veces por semana); y Grupo Comunidad, integrado por personas de la comunidad que manifestaron interés en participar, que no realizaban actividad física habitualmente.

La investigación contó con la aprobación del Comité de ética de la Universidad Autónoma de Chile.

\section{Procedimiento}

Los participantes fueron asignados de acuerdo a los perfiles en tres grupos. Una vez entregada la información del estudio y firmado el consentimiento informado, se constataron sus antecedentes de salud, confirmando que cumplieran los criterios de inclusión. Solo después, se aplicaron individualmente los test neuropsicológicos, en dos sesiones.

En la primera sesión se aplicó el Test de inteligencia de Kaufman (K-BIT; 1996) y el Minimental State versión EFAM-Chile (1999) con el objetivo de homogenizar el cociente intelectual 
y descartar el deterioro cognitivo en los sujetos. Posteriormente, se realizó el test de capacidad física en adulto mayor o VACAFUN, que mide la capacidad funcional en adultos mayores y consta de mediciones en fuerza, flexibilidad y capacidad aeróbica. Por último, se realizó el Cuestionario de Reserva cognitiva (CRC) propuesto por Rami et al (2011). El CRC está formado por 24 ítems distribuidos en cuatro áreas: Actividades de la Vida Diaria, Formación, Aficiones y Vida Social.

En la segunda sesión se aplicó el ANTI-V, realizado en una habitación acondicionada, controlando las condiciones ambientales (ruido, iluminación, temperatura). Los sujetos fueron evaluados individualmente por el profesional kinesiólogo a cargo de la investigación. Los estímulos se mostraron en un monitor CTR de 17 pulgadas, con resolución de pantalla de 640 X480 píxeles, además se utilizó auriculares para presentar los sonidos de alerta. Las respuestas se recogieron a través del teclado de ordenador.

La descripción de la tarea ANTI-V se detalla en el estudio de Martella et al (2014). En líneas generales, la tarea se divide en dos bloques. El primer bloque (ANTI) consta de 20 ensayos de práctica, seguidos de 48 experimentales, midiendo la eficiencia de las redes atencionales. El segundo bloque (ANTI-V) comprende 20 ensayos práctica, seguidos de 192 experimentales presentando aleatoriamente la condición vigilancia.
Respecto al procedimiento, se presentaba una pantalla inicial compuesta por una cruz central y una fila de boxes arriba y debajo, con duración variable entre 1.200 y $2.600 \mathrm{~ms}$, después de la cual se emitía un tono de alerta auditiva en la mitad de los ensayos y durante $50 \mathrm{~ms}$ (condición de alerta fásica). La señal visual aparecía $350 \mathrm{~ms}$ después del tono y se presentaba por $50 \mathrm{~ms}$ en el box central de la fila superior o inferior. El estímulo objetivo (target) y los flancos (flechas) podían aparecer en la misma fila que la señal (50\% de los ensayos; condición válida), o en la otra fila (50\% de los ensayos; condición inválida). Finalmente, las flechas podían apuntar en la misma dirección del objetivo en la mitad de los ensayos (condición congruente), o hacia la dirección opuesta del objetivo en la otra mitad (condición incongruente).

Por último, la flecha central aparecía más corta en $25 \%$ de los ensayos (Bloque ANTI-V). Se animó a los participantes a identificar los estímulos infrecuentes presionando la barra espaciadora, ignorando la dirección de la flecha central en estos ensayos. Los participantes realizaron primero el bloque ANTI, con un descanso de $5 \mathrm{~min}$ y luego realizaron el bloque ANTI-V sin descanso adicional. La Figura 1 muestra los estímulos y procedimientos de la tarea.

\section{Análisis estadístico}

Los datos se analizaron con el software estadís-

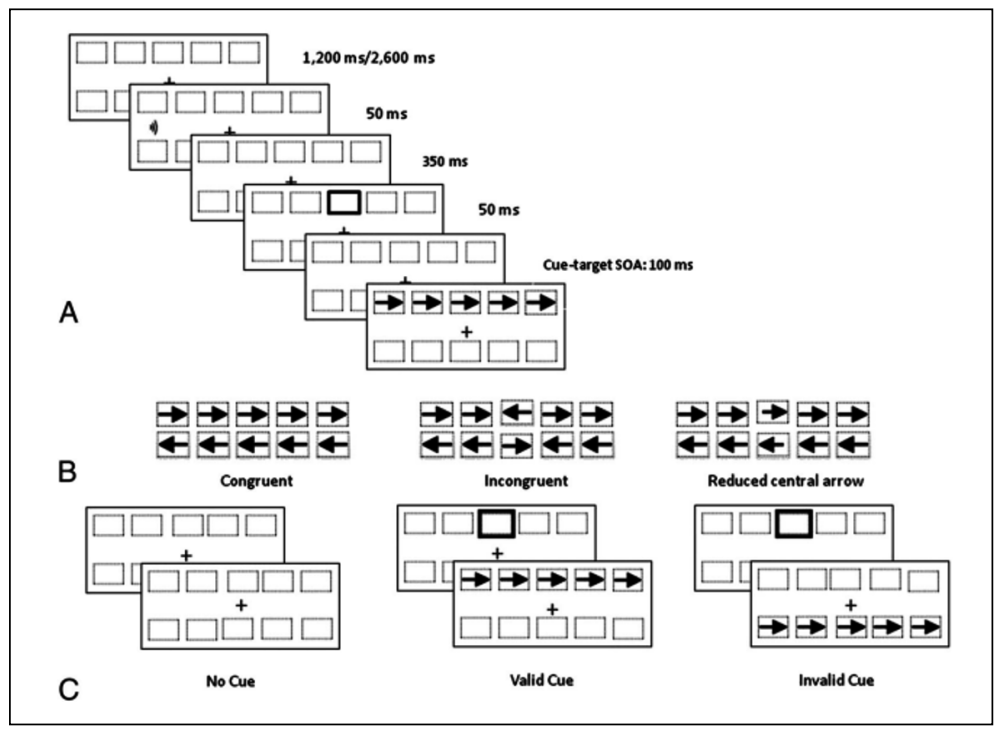

Figura 1. Procedimiento y estímulos utilizados en ANTI-V (Martella et al., 2014). A. Representación esquemática del procedimiento. B. Estímulos objetivo. C. Condiciones de señal visual. 
tico IBM SPSS 22 (2013) y Microsoft Excel (2014). Los resultados de las características sociodemográficas, test neuropsicológicos y funcionales de los grupos se reportan en la Tabla 1.

Se analizó el promedio en tiempos de respuesta y porcentaje de aciertos de los ensayos correctos del ANTI-V. Los filtros para tiempos de respuestas fueron de 200 y $1.700 \mathrm{~ms}$, descartando respuestas anticipadas u omisiones. Posteriormente se realizó un ANOVA mixto con factor independiente por Grupo (Artrosis, Actividad física y Comunidad) $\mathrm{x}$ Tono de alerta (presente, ausente) x Señal (válido, inválida, sin señal) x Congruencia (congruente, incongruente).

En cuanto a la tarea de vigilancia, se calcularon los hits (respuestas correctas al estímulo infrecuente) y las falsas alarmas (respuestas incorrectas al estímulo infrecuente) obteniendo el índice de sensibilidad (d') y el criterio de respuesta $(\beta)$, siguiendo el procedimiento de la TDS. Los índices se sometieron a ANOVA unidireccional con el factor independiente Grupo.

\section{Resultados}

\section{Tiempos de respuesta}

El ANOVA indicó un efecto significativo de la alerta $\left(F(1,36)=38,20, p=<0,000001 ; \eta^{2}=0,40\right)$,la orientación $\left(\mathrm{F}(2,72)=4,78, \mathrm{p}=0,01 ; \eta^{2}=0,077\right)$ y la atención ejecutiva $(\mathrm{F}(1,36)=27,66, \mathrm{p}=0,000002$; $\left.\eta^{2}=0,33\right)$.

Los tiempos de respuesta fueron más rápidos con tono de alerta $(615,38 \mathrm{~ms})$ respecto a cuándo el tono era ausente $(656,56 \mathrm{~ms})$; el tiempo de respuesta fue menor en las condiciones de señal válida $(609,81 \mathrm{~ms})$ que en condición de señal inválida y sin señal $(652,73$ ms y 645,35 ms, respectivamente); también se encontró un mayor tiempo de respuesta en la condición incongruente $(664,73 \mathrm{~ms})$ respecto a la condición congruente $(607,21 \mathrm{~ms})$.

Ningún otro efecto o interacción resultaron significativos.

\section{Porcentajes de errores}

El análisis indicó un efecto significativo de la alerta $\left(\mathrm{F}(1,36)=4,38, \mathrm{p}=0,04 ; \eta^{2}=0,07\right)$, la orientación $\left(\mathrm{F}(2,72)=31,51, \mathrm{p}<0,000001 ; \eta^{2}=0,36\right)$ y la atencional ejecutiva $(\mathrm{F}(1,36)=36,99, \mathrm{p}<0,000001$; $\left.\eta^{2}=0,39\right)$.

Ningún otro efecto o interacción resultaron significativos.

\section{Análisis de la vigilancia}

El ANOVA para la comparación de los índices del bloque vigilancia en la prueba ANTI-V no

Tabla 1. Información Sociodemográfica, promedios ( $(\mathrm{SD})$ en test neuropsicológicos y funcionales para los grupos de participantes

\begin{tabular}{|c|c|c|c|c|c|c|}
\hline \multirow{2}{*}{$\begin{array}{l}\text { Datos sociodemógráficos y tests } \\
\mathrm{n}\end{array}$} & \multicolumn{2}{|c|}{ Artrosis } & \multicolumn{2}{|c|}{ Actividad física } & \multicolumn{2}{|c|}{ Comunidad } \\
\hline & 20 & & 20 & & 20 & \\
\hline Sexo: femenino/masculino & $17 / 3$ & & $17 / 3$ & & $18 / 2$ & \\
\hline Edad, años & 70,95 & $(4,42)$ & 68,55 & $(3,25)$ & 70,5 & $(6,35)$ \\
\hline \multicolumn{7}{|l|}{ Educación } \\
\hline Primaria & 12 & & 10 & & 10 & \\
\hline Secundaria/universitaria & $8 / 0$ & & 9/1 & & $8 / 2$ & \\
\hline MMSE $^{a}$ & 18,10 & $(1,25)$ & 18,70 & $(0,57)$ & 18,85 & $(0,36)$ \\
\hline$C R C^{b}$ & 6,95 & $(2,28)$ & 8,85 & $(3,26)$ & 8,90 & $(4,47)$ \\
\hline $\mathrm{K}-\mathrm{BIT}$ & 116,60 & $(28,87)$ & 110,30 & $(19,47)$ & 116,45 & $(22,87)$ \\
\hline \multicolumn{7}{|l|}{ VACAFUN } \\
\hline Test 1. Sentarse y levantarse de silla (rep) & 15,25 & $(3,46)$ & 16,75 & $(3,29)$ & 14,65 & $(2,77)$ \\
\hline Test 2. Flexiones de brazo (rep) & 25,00 & $(6,48)$ & 29,30 & $(4,85)$ & 24,85 & $(5,27)$ \\
\hline Test 3. Flexión de tronco en silla $(\mathrm{cm})$ & 0,51 & $(7,45)$ & 4,30 & $(4,06)$ & 1,40 & $(2,39)$ \\
\hline Test 4. Juntar las manos tras la espalda (cm) & $-9,40$ & $(11,86)$ & $-9,25$ & $(11,86)$ & $-5,7$ & $(2,54)$ \\
\hline Test 5. TUG (seg) & 8,56 & $(2,54)$ & 7,06 & $(1,30)$ & 6,95 & $(0,97)$ \\
\hline Test 6. Caminar 6 min (mts) & 328,2 & $(95,97)$ & 489,4 & $(71,58)$ & 449,65 & $(84,08)$ \\
\hline
\end{tabular}

aMinimental State, ${ }^{b}$ Cuestionario de reserva cognitiva, ${ }^{\mathrm{C}}$ Test de inteligencia de Kaufman. 
Factores protectores de funciones atencionales en adultos mayores - G. Wikee et al

Tabla 2. Promedio ( \pm DS) de los tiempos de respuesta y porcentaje de aciertos en función de las condiciones del ANTI-V y de los grupos

\begin{tabular}{|c|c|c|c|c|c|c|c|c|c|c|c|c|}
\hline & \multicolumn{4}{|c|}{ Grupo Artrosis } & \multicolumn{4}{|c|}{ Grupo Actividad física } & \multicolumn{4}{|c|}{ Grupo Comunidad } \\
\hline & \multicolumn{2}{|c|}{ Tono } & \multicolumn{2}{|c|}{ No tono } & \multicolumn{2}{|c|}{ Tono } & \multicolumn{2}{|c|}{ No tono } & \multicolumn{2}{|c|}{ Tono } & \multicolumn{2}{|c|}{ No tono } \\
\hline & $\begin{array}{l}\text { Pro- } \\
\text { medio }\end{array}$ & DS & $\begin{array}{l}\text { Pro- } \\
\text { medio }\end{array}$ & DS & $\begin{array}{l}\text { Pro- } \\
\text { medio }\end{array}$ & DS & $\begin{array}{l}\text { Pro- } \\
\text { medio }\end{array}$ & DS & $\begin{array}{l}\text { Pro- } \\
\text { medio }\end{array}$ & DS & $\begin{array}{l}\text { Pro- } \\
\text { medio }\end{array}$ & DS \\
\hline \multicolumn{13}{|c|}{ Tiempo de respuesta (ms) } \\
\hline Valido congruente & 532,24 & 198,83 & 592,52 & 269,60 & 619,94 & 229,88 & 659,47 & 204,40 & 548,77 & 178,53 & 792,35 & 408,98 \\
\hline Incongruente & 577,54 & 219,51 & 615,84 & 220,55 & 676,86 & 214,96 & 723,00 & 264,03 & 641,21 & 312,56 & 829,87 & 396,21 \\
\hline Invalido congruente & 575,58 & 257,02 & 548,77 & 178,53 & 665,33 & 180,08 & 697,35 & 213,42 & 583,95 & 198,63 & 763,14 & 257,77 \\
\hline Incongruente & 598,92 & 269,11 & 641,21 & 312,56 & 751,17 & 266,98 & 826,61 & 239,26 & 641,75 & 191,24 & 867,79 & 379,59 \\
\hline Sin señal congruente & 551,54 & 221,70 & 583,95 & 198,63 & 618,95 & 162,11 & 683,04 & 199,65 & 798,07 & 436,32 & 819,73 & 377,42 \\
\hline Incongruente & 566,73 & 202,00 & 641,75 & 191,24 & 690,77 & 206,94 & 811,22 & 284,56 & 736,52 & 412,19 & 825,42 & 238,69 \\
\hline \multicolumn{13}{|l|}{ Aciertos (\%) } \\
\hline Valido congruente & 0,26 & 0,28 & 0,25 & 0,24 & 0,23 & 0,21 & 0,23 & 0,20 & 0,08 & 0,09 & 0,12 & 0,11 \\
\hline Incongruente & 0,36 & 0,28 & 0,45 & 0,31 & 0,34 & 0,28 & 0,32 & 0,23 & 0,22 & 0,28 & 0,23 & 0,26 \\
\hline Invalido congruente & 0,34 & 0,22 & 0,36 & 0,23 & 0,34 & 0,17 & 0,31 & 0,20 & 0,15 & 0,12 & 0,23 & 0,14 \\
\hline Incongruente & 0,48 & 0,24 & 0,49 & 0,24 & 0,50 & 0,31 & 0,47 & 0,24 & 0,38 & 0,23 & 0,39 & 0,27 \\
\hline Sin señal congruente & 0,28 & 0,23 & 0,32 & 0,20 & 0,20 & 0,18 & 0,30 & 0,22 & 0,10 & 0,07 & 0,13 & 0,11 \\
\hline Incongruente & 0,35 & 0,23 & 0,46 & 0,26 & 0,35 & 0,29 & 0,43 & 0,28 & 0,26 & 0,20 & 0,30 & 0,25 \\
\hline
\end{tabular}

reportó diferencias significativas entre los grupos de estudio.

En cuanto al análisis de TDS, el promedio del índice de sensibilidad (d') fue menor para el grupo Artrosis $(\mathrm{M}=1,08)$ que los grupos de Actividad física $(M=1,28)$ y Comunidad $(M=1,27)$, pero no se observó diferencias significativas en el criterio de respuesta $\beta$, y d' $(F<1)$.

\section{Discusión}

La reserva cognitiva y la actividad física se consideran factores protectores del deterioro cognitivo en la tercera edad ${ }^{19-27}$. Por otro lado, hay estudios que indican que la atención es la primera función cognitiva en empeorar y puede ser clave en la detección precoz de demencia y en establecer un plan de intervención cognitiva en adultos mayores ${ }^{1,6-11}$.

Respecto a los datos sociodemográficos y neuropsicológicos (sexo, edad, escolaridad, MMSE, K-BIT y CRC), no se evidenciaron diferencias, sino en la capacidad física, medida con la batería VACAFUN.

Los grupos mantuvieron un nivel homogéneo en el CRC, limitando las comparaciones de éste indicador. Este instrumento, validado para la población española, objetiva el grado de reserva cognitiva, siendo utilizado en la práctica clínica e investigaciones ${ }^{26,27}$. El carácter retrospectivo de la RC, limita la posibilidad de tener evidencia anatomo-patológica cerebral en personas vivas, impidiendo la interpretación y síntesis clara de los hallazgos en las investigaciones ${ }^{24}$. En este estudio el cuestionario fue utilizado de forma experimental, sin relacionar los resultados con la población española.

En relación a la capacidad física, se debe considerar que el grupo Artrosis participa en entrenamiento grupal que incluye ejercicio aeróbico, fortalecimiento muscular y dinámicas gerontológicas de socialización. Este grupo mostró un menor desempeño físico, situación inherente a su condición de salud, no obstante, los test neuropsicológicos y pruebas atencionales no se vieron afectadas. Existe abundante evidencia sobre los beneficios del ejercicio físico, donde los aspectos de la cognición, son inseparables de la locomoción y la actividad física ${ }^{22}$. La actividad cardiovascular ${ }^{31,32}$ y el ejercicio aeróbico pueden mejorar la plasticidad cerebral y reducir la senescencia biológica en el envejecimiento humano ${ }^{20,22,23}$. Sin embargo, 
no está suficientemente documentado que tipo, intensidad o frecuencia de ejercicio es más eficaz, ni la complementariedad con otras técnicas de intervención ${ }^{20}$.

Respecto a las redes atencionales, resultaron significativos los efectos principales relativos a Alerta, Orientación y Congruencia, indicando que el ANTI-V puede ser un instrumento eficaz en la medición del funcionamiento atencional, confirmando los resultados de otros estudios ${ }^{7,8,19,32,33}$. Además, al no evidenciar diferencias entre grupos en el ANTI-V y RC, los resultados indican que no hay diferencia en la función atencional cuando hay diferentes niveles de actividad física, en contraste con el grupo Comunidad, donde la presencia del estímulo sonoro indujo un menor efecto de alerta. Estudios previos demuestran que el ejercicio es una actividad "excitante" que estimula la secreción de catecolaminas ${ }^{12,13,36}$, consecuentemente, se esperaba que la función de alerta fuera modulada por el efecto protector de la actividad física, situación que concordó con la literatura. No obstante, datos previos muestran resultados contradictorios a corto plazo ${ }^{32}$.

La red atencional de orientación se demostró funcional en los tres grupos, resultado que está en línea con estudios previos, indicando que el ejercicio puede aumentar la flexibilidad en el desplazamiento del foco atencional en el espacio ${ }^{32,37}$. Por último, no se observaron diferencias en la red atencional ejecutiva. No existen investigaciones que comparen el efecto del ejercicio crónico sobre la función atencional ejecutiva en adultos mayores.

Otro aspecto relevante, es el análisis de la vigilancia mediante la TDS, que reportó un menor desempeño en el grupo Artrosis, con mayor porcentaje de error, un menor índice de sensibilidad (d') y criterio de respuesta $(\beta)$. La evidencia previa es limitada, pero un estudio que incluye la prueba de vigilancia, demostró aportar una medición directa del déficit de alerta tónica en adultos con deterioro cognitivo leve $e^{8}$, sin embargo, se necesita un mayor número de participantes con diferentes habilidades motoras para aclarar el efecto del ejercicio físico sobre la vigilancia. Este resultado es relevante, considerando que los participantes no presentaban deterioro cognitivo. Dentro de los alcances, sería recomendable incorporar un grupo de adultos mayores sedentarios, que permita contrastar los cambios cognitivos asociados a ésta condición.
Finalmente, los resultados de nuestro estudio confirman el efecto protector de la actividad física permanente, que independientemente del tipo o intensidad, favorece el funcionamiento atencional, convirtiéndose en una estrategia psicosocial prometedora en el envejecimiento cognitivo. La reserva cognitiva, como factor, aún requiere mejorar las estrategias metodológicas para obtener resultados concluyentes.

En conclusión, los factores protectores expuestos, al igual que la medición de la atención, representan una estrategia eficaz para su aplicación en medicina demostrando ser una alternativa viable y de amplia utilidad en la promoción de salud con adultos mayores, siendo un componente fundamental en los programas terapéuticos y de estimulación cognitiva implementados a nivel nacional.

Los hallazgos presentados, junto con investigación futura, aportan al conocimiento sobre aspectos específicos de la atención, relevantes para la aplicación terapéutica y el diagnóstico precoz en adultos mayores con deterioro cognitivo y etapas iniciales de EA.

Reconocimiento: Esta tesis es parte del trabajo final de magíster de German Wikee, adscrito al programa de post grado en Neurociencias de la Universidad Autónoma de Chile, sede Santiago.

\section{Referencias}

1. Fernández P, Campoy G, García Santos J, Antequera M, García-Sevilla J, Castillo A, et al. Is there a specific pattern of attention deficit in mild cognitive impairment with subcortical vascular features? Evidence from the attention network test. Dement Geriatr Cogn Disord 2011; 31 (4): 268-75. Disponible en: www.karger.com [Consultado el 12 de junio de 2017].

2. Petersen R, Doody R, Kurz A, Mohs R, Morris J, Rabins $\mathrm{P}$, et al. Current Concepts in Mild Cognitive Impairment. Arch Neurol 2001; 58(12):1985-92. Disponible en https://jamanetwork.com [Consultado el 12 de junio de 2017].

3. Sánchez-Rodríguez J, Torrellas-Morales C. Revisión del constructo deterioro cognitivo leve: Aspectos generales. Rev Neurol 2011; 52 (5): 300-5. Disponible en: www. neurologia.com [Consultado el 21 de julio de 2016].

4. Hwang H, Choi S, Yoon D, Yoon B, Suh Y, Lee D, et al. The effect of cognitive training in patients with mild 
cognitive impairment and early Alzheimer's disease: a preliminary study. J Clin Neurol 2012; 8 (3): 190-7. Disponible en: www.thejcn.com [Consultado el 12 de junio de 2017].

5. Aretouli E, Tsilidis K, Brandt J. Four-year outcome of mild cognitive impairment: The contribution of executive dysfunction. Neuropsychology 2013; 27 (1): 95-106. Disponible en: www.apa.org [Consultado el 12 de junio de 2017].

6. Fuentes L, Campoy G. The time course of alerting effect over orienting in the attention network test. Exp Brain Res 2008; 185 (4): 667-72. Disponible en: https://link. springer.com [Consultado el 12 de junio de 2017].

7. Martella D, Manzanares S, Campoy G, Roca J, Antúnez C, Fuentes L. Phasic and tonic alerting in mild cognitive impairment: A preliminary study. Exp Gerontol 2014; 49 (1): 35-9. Disponible en: www.sciencedirect.com [Consultado el 21 de julio de 2016].

8. Saunders N, Summers M. Longitudinal deficits to attention, executive, and working memory in subtypes of mild cognitive impairment. Neuropsychology 2011; 25 (2): 237-48. Disponible en: www.apa.org [Consultado el 12 de junio de 2017].

9. Tales A, Snowden R, Phillips M, Haworth J, Porter G, Wilcock G, et al. Exogenous phasic alerting and spatial orienting in mild cognitive impairment compared to healthy ageing: Study outcome is related to target response. Cortex 2011; 47 (2): 180-90. Disponible en: www.sciencedirect.com [Consultado el 12 de junio de 2017].

10. Van Dam N, Sano M, Mitsis E, Grossman H, Gu X, Park $\mathrm{Y}$, et al. Functional Neural Correlates of Attentional Deficits in Amnestic Mild Cognitive Impairment. Plos One 2013; 8 (1). Disponible en: journals.plos.org [Consultado el 12 de junio de 2017].

11. Petersen S, Posner M. The attention system of the human brain. Annu Rev Neurosci 1990; 13: 25-42. Disponible en: www.annualreviews.org [Consultado el 12 de junio de 2017].

12. Posner M. Orienting of attention. Q J Exp Psychol 1980; 32 (1): 3-25. Disponible en: www.ncbi.nlm.nih.gov [Consultado el 12 de junio de 2017].

13. Vázquez-Marrufo M, Benítez M, Rodríguez-Gómez G, Galvao-Carmona A, Fernández-Del Olmo A, Vaquero-Casares E. Afectación de las redes neurales atencionales durante el envejecimiento saludable. Rev Neurol 2011; 52 (1): 20-6. Disponible en: www.neurologia.com [Consultado el 12 de junio de 2017].

14. Fan J, McCandliss B, Sommer T, Raz A, Posner M. Testing the efficiency and independence of attentional networks. J Cogn Neurosci 2002; 14 (3): 340-7. Dispo- nible en: www.mitpressjournals.org/jocn [Consultado el 21 de julio de 2016].

15. Callejas A, Lupiáñez J, Tudela P. The three attentional networks: On their independence and interactions. Brain Cogn 2004; 54 (3): 225-7. Disponible en: www. sciencedirect.com [Consultado el 12 de junio de 2017].

16. Roca J, Castro C, López-Ramón M, Lupiáñez J. Measuring vigilance while assessing the functioning of the three attentional networks: The ANTI-Vigilance task. J Neurosci Methods 2011; 198 (2): 312-24. Disponible en: www.sciencedirect.com [Consultado el 12 de junio de 2017].

17. Fan J, McCandliss B, Fossella J, Flombaum J, Posner M. The activation of attentional networks. NeuroImage 2005; 26 (2): 471-9. Disponible en: www.sciencedirect. com [Consultado el 12 de junio de 2017].

18. Fernández-Duque D, Black S. Attentional networks in normal aging and Alzheimer's disease. Neuropsychology 2006; 20 (2): 133-43. Disponible en: www.apa.org [Consultado el 12 de junio de 2017].

19. Franco-Martin M, Parra-Vidales E, González-Palau F, Bernate-Navarro M, Solis A. Influencia del ejercicio físico en la prevención del deterioro cognitivo en las personas mayores: revisión sistemática. Rev Neurol 2013; 56 (11): 545-54. Disponible en: www.neurologia. com [Consultado el 21 de julio de 2016].

20. Salech M, Jara L. Physiological changes associated with normal aging. Rev Med Clin Condes 2012; 23(1): 19-29. Disponible en: www.clc.cl [Consultado el 12 de junio de 2017].

21. Kempermann G, Fabel K, Ehninger D, Babu H, Leal-Galicia P, Garthe A, et al. Why and how physical activity promotes experience-induced brain plasticity. Front Neurosci 2010; 4: 1-9. Disponible en: www.frontiersin. org [Consultado el 21 de julio de 2016].

22. Riquelme D, Sepúlveda C, Muñoz M, Valenzuela M. Ejercicio físico y su influencia en los procesos cognitivos. Revista Motricidad y Persona 2013; (13): 69-74. Disponible en: https://dialnet.unirioja.es [Consultado el 21 de julio de 2016].

23. Lojo-Seoane C, Facal D, Juncos-Rabadín O. Previene la actividad intelectual el deterioro cognitivo? Relaciones entre reserva cognitiva y deterioro cognitivo ligero. Rev Esp Geriatr Gerontol 2012; 47(6): 270-8. Disponible en: www.elsevier.es [Consultado el 12 de junio de 2017].

24. Díaz-Orueta U, Buiza-Bueno C, Yanguas-Lezaun J. Reserva cognitiva: evidencias, limitaciones y líneas de investigación futura. Rev Esp Geriatr Gerontol 2012; 45 (3): 150-5. Disponible en: www.elsevier.es [Consultado el 21 de julio de 2016].

25. Rami L, Valls-Pedret C, Bartrés-Faz D, Caprile C, So- 
lé-Padullés C, Castellví M, et al. Cuestionario de reserva cognitiva. Valores obtenidos en población anciana sana y con enfermedad de Alzheimer. Rev Neurol 2011; 52 (4): 195-201. Disponible en: www.neurologia.com [Consultado el 12 de junio de 2017].

26. Rodríguez M, Sánchez J. Reserva cognitiva y demencia. An Psicol 2004; 20 (2000): 175-86. Disponible en: http:// scielo.isciii.es [Consultado el 21 de julio de 2016].

27. La Rue A. Healthy brain aging: role of cognitive reserve, cognitive stimulation, and cognitive exercises. Clin Geriatr Med 2010; 26 (1): 99-111. Disponible en: www. geriatric.theclinics.com [Consultado el 21 de julio de 2016].

28. Ato M, López J. Un sistema de clasificación de los diseños de investigación en psicología. An Psicol 2013; 29 (3): 1038-59. Disponible en: http://scielo.isciii.es [Consultado el 26 de agosto de 2016].

29. Carbonel A, Aparicio V, Delgado M. Effects of aging on physical fitness: implications in the recommendations of physical activity for older adults. Rev Int Cienc Deporte 2009; 5 (17): 1-18. Disponible en: www.cafyd.com [Consultado el 12 de junio de 2017].

30. Colcombe S, Kramer A, Erickson K, Scalf P, McAuley E, Cohen N, et al. Cardiovascular fitness, cortical plasticity, and aging. Proc Natl Acad Sci 2004; 101 (9): 3316-21. Disponible en: www.pnas.org [Consultado el 13 de junio de 2017].

31. Huertas F, Zahonero J, Sanabria D. Functioning of the
Attentional Networks at Rest vs. During Acute Bouts of Aerobic Exercise. J Sport Exerc Psychol 2011; 33: 649-65. Disponible en: journals.humankinetics.com [Consultado el 13 de junio de 2017].

32. Mahoney J, Verghese J, Goldin Y, Lipton R. Alerting, orienting, and executive attention in older adults. J Int Neuropsychol Soc 2011; 16 (5): 877-89. Disponible en: www.cambridge.org [Consultado el 12 de junio de 2017].

33. Jennings J, Dagenbach D, Engle C, Funke L. Age-Related Changes and the Attention Network Task: An Examination of Alerting, Orienting, and Executive Function. Aging Neuropsychol Cogn 2007; 14 (4): 353-69. Disponible en: www.tandfonline.com [Consultado el 12 de junio de 2017].

34. Liu-Ambrose T, Nagamatsu L, Graf P, Beattie B, Ashe M, Handy T. Resistance Training and Executive Functions: A 12-Month Randomised Controlled Trial. Arch Intern Med 2012; 170 (2): 170-8. Disponible en: jamanetwork.com [Consultado el 12 de junio de 2017].

35. Tomporowski P. Effects of acute bouts of exercise on cognition. Acta Psychol 2003; 112 (3): 297-324. Disponible en: www.journals.elsevier.com [Consultado el 12 de junio de 2017].

36. Colmenero J, Catena A, Fuentes L. Atención visual: una revisión sobre las redes atencionales del cerebro. An Psicol 2001; 17 (1): 45-67. Disponible en: http://scielo. isciii.es [Consultado el 21 de julio de 2016]. 